Silvia

Bou Ysàs*

Magda

Cayón Costa ***

Albert

Hernández ${ }^{* * * * *}$

Universidad Autónoma de Barcelona, Barcelona, España

Recibido: 15 de octubre de 2013 Concepto de evaluación: 5 de febrero de 2014 Aprobado: 17 de marzo de 2014

Artículo de reflexión DOI: http://dx.doi.org/10.14718/ revfinanzpolitecon.2014.6.1.4

(C) 2014 Universidad Católica de Colombia. Facultad de Ciencias Económicas y Administrativas. Todos los derechos reservados.

*Doctora en Ciencias Económicas, magíster en Economía, Economista. Docente de la Facultad de Economía y Empresa, Universidad Autónoma de Barcelona, Barcelona, España. Correo electrónico: silvia.bou@uab.cat

**Doctora en Ciencias Económicas, magíster en Economía, Economista. Docente de la Facultad de Economía y Empresa, Universidad Autónoma de Barcelona, España.

Correo electrónico: magda.cayon@uab.cat

*** Licenciado en Ciencias Económicas. Banco de Sabadell, Madrid, España. Correo electrónico: albert.hernandezco@ campus.uab.es

Dirección de correspondencia autores: Departamento de Empresa, Edificio B, Campus de Bellaterra, 08193 Cerdanyola del Vallès, Barcelona, España.

\section{Análisis de la heurística en la contratación empresarial a través de una cartera de derivados reales}

\section{RESUMEN}

Dada la situación actual y partiendo del supuesto que como herramienta para una correcta política de empleo la legislación laboral debe sentar las bases sólidas para que en el momento de recuperación económica se generen puestos de trabajo estables, se presenta un modelo de simulación de contratación que nos va a permitir un análisis de sensibilidad de las distintas variables a tener en cuenta en una regulación del mercado laboral.

El modelo consiste en asimilar el comportamiento del empresario al del propietario de una cartera de inversión compuesta por dos derivados reales, un swap y una opción de venta americana. Este modelo nos permite, por un lado, hacer un análisis de sensibilidad de las diferentes variables que intervienen en la contratación, y, por otro lado, analizar los efectos que la última reforma laboral española tiene sobre la decisión de contratar del empresario. Los resultados son claros, la variable que más sensibilidad presenta sobre la contratación es la cuota patronal de la seguridad social, y aplicando los cambios propuestos en esta última reforma laboral, se concretan estos efectos y también se concluye que la reducción de los costes de despido no acelera la decisión de contratar.

Palabras clave: demanda laboral, contrato laboral, derivados reales, modelo de simulación.

JEL: J23, J41, G13, C53

\section{Analysis of Heuristics in Business Recruitment based on a Portfolio of Real Derivatives}

\section{ABSTRACT}

Departing from the notion that labour legislation should be founded in the generation of job stability a model for recruitment is presented in which the employer is likened to the holder of an investment portfolio containing two real derivatives - swap or the option of sale. This model allows us on one hand to analyze sensitivity to the variables that are at play in an employment contract and, on the other, look at the effects that the most recent reforms in Spanish labour laws have had on contracting decisions made by employers. The results are clear: social security benefits are shown to be the most sensitive variable on the work contract, and applying the changes proposed in the latest labour reforms, this effect is upheld. The study concludes that reducing the costs of dismissal does not increase the likelihood of employers' taking on new staff.

Keywords: Labour demand, work contract, real derivatives, simulation model. 


\section{Análise da heurística na contratação empresarial por meio de uma carteira de derivados reais}

\section{RESUMO}

Dada a situação atual e partindo do suposto que como ferramenta para uma correta política de emprego, a legislação laboral deve instituir as bases sólidas para que, no momento da recuperação econômica, se gerem postos de trabalho estáveis, se apresentem um modelo de simulação de contratação que nos permitirá uma análise de sensibilidade das diferentes variáveis a considerar em uma regulação do mercado laboral.

O modelo consiste em assimilar o comportamento do empresário ao do proprietário de uma carteira de investimento composto por dois derivados reais, um swap e uma opção de venda americana. Esse modelo nos permite, por um lado, fazer uma análise de sensibilidade das diferentes variáveis que intervêm na contratação e, por outro lado, analisar os efeitos que a última reforma laboral espanhola tem sobre a decisão de contratar do empresário. Os resultados são claros, a variável que mais sensibilidade apresenta sobre a contratação é a cota patronal da previdência social e aplicando as mudanças propostas nesta última reforma laboral, concretizam-se esses efeitos e também se conclui que a redução dos custos de demissão não acelera a decisão de contratar.

Palavras-chave: demanda laboral, contrato laboral, derivados reais, modelo de simulação. 


\section{INTRODUCCIÓN}

La situación económica actual ha incitado el cuestionamiento hacia los modelos productivos actuales. La desconfianza en los mercados y las inciertas previsiones provocan que los empresarios hayan tomado decisiones de austeridad en sus presupuestos. Los recortes empresariales han implicado reajustes en la producción que se han traducido en numerosas rescisiones de contratos. Uno de los temas que se plantean los políticos es cómo la legislación puede ayudar al empresario y trabajadores en estos momentos de incertidumbre económica, para flexibilizar el mercado laboral.

Si analizamos la historia más reciente, el mercado laboral español ha experimentado seis importantes reformas desde la aprobación de la Constitución española en 1978, de la cual surgió el Estatuto de los Trabajadores. La primera se produjo en 1984, y su objetivo era reducir el elevado nivel de desempleo a través del impulso de la contratación temporal creando el contrato de fomento al empleo y el contrato eventual por circunstancias de producción, ampliando el contrato en prácticas, el de tiempo parcial y el de lanzamiento de nueva actividad. También se reformó la protección por desempleo. Esta reforma incrementó el número de ocupados, pero generó una alta tasa de temporalidad y no consiguió corregir la tasa de paro, que en 1993 era del $22,7 \%$.

La segunda reforma se produjo en 1994 y fue calificada, según Sandalio Gómez ${ }^{1}$, como la reforma más importante del Estatuto de los Trabajadores. Su principal objetivo era reducir la contratación temporal suprimiendo el contrato de fomento al empleo, y facilitar la inserción juvenil impulsando los contratos de formación (prácticas y aprendizaje). La pretensión era flexibilizar el desarrollo de la relación laboral y el despido individual y colectivo, y de esta manera ampliar las posibilidades del despido objetivo. Según el Anuario de Estadísticas Laborales del Ministerio de Trabajo y Asuntos Sociales, desde 1994 hasta 1997 los contratos temporales incrementaron el $7 \%$, y

1 En su estudio Las reformas laborales en España y su impacto real en el mercado de trabajo en el período 1985-2008. fracasaron en uno de los principales objetivos. En cambio se consiguió reducir sensiblemente la tasa de paro hasta situarse en el $20,8 \%$. Pero dicha reforma dejó insatisfechos tanto a los sindicatos, como a los empresarios.

La tercera reforma de 1996 nació del acuerdo entre las dos principales patronales CEOE y Cepyme, y los sindicatos mayoritarios CCOO y UGT. Su principal objetivo fue incrementar la contratación indefinida y para ello se creó el contrato de fomento de la contratación indefinida, con una indemnización menor en el caso de despido objetivo declarado improcedente (33 días por año de servicio, con un tope de dos años). Para reducir la temporalidad en los contratos se eliminó el contrato por lanzamiento de nueva actividad y se modificaron las condiciones del contrato de formación y del contrato en prácticas. Según los datos oficiales se consiguió reducir la proporción de contratos temporales en un $2 \%$ entre 1998 y 2001, y también se redujo notablemente la tasa de paro, siendo en 2001 del 10,5\%.

A pesar de la buena situación del mercado laboral del final de la década de los 90 y del buen clima social dado por el consenso entre los agentes sociales, se llevó a cabo la cuarta reforma laboral que se aprobó en 2001 sin consenso y de forma unilateral por el Gobierno. Esta reforma pretendió ampliar el horizonte temporal de las medidas tomadas en 1997 para aumentar los beneficios que supuestamente estaba obteniendo el mercado laboral español. Así mismo, se fomentó la contratación a tiempo parcial, se redujo a 12 meses la duración máxima de los contratos eventuales por causas de producción, se creó el contrato de inserción (contrato temporal para el personal de Administración Pública), se abarató el despido para los contratos fijos y se encareció el de los contratos temporales para estimular la conversión de contratos temporales en indefinidos. A pesar de las medidas aprobadas, en términos absolutos los contratos temporales aumentaron un $13,4 \%$, pero la contratación indefinida también aumentó un $14,5 \%$ y la tasa de paro se redujo al situarse en 2006 en el $8,5 \%$ de la población activa. 
En 2006 llegó un acuerdo de Gobierno, patronal y sindicatos para una nueva reforma laboral que quería combatir la contratación temporal y rebajar la tasa de desempleo, que era las más alta de toda Europa. La pretensión era rebajar los costes laborales de las empresas y mejorar la protección de desempleo de colectivos específicos. Para ello se bonificó y estimuló la contratación indefinida, la conversión de temporales en fijos y se redujeron las cotizaciones empresariales al Fondo de Garantía Salarial y por desempleo. Resulta imposible evaluar los resultados de esta reforma ya que a finales de 2008 el país entró técnicamente en recesión debido a la actual crisis económica. Un hecho es que la tasa de paro pasó del 7,95\% en 2007 al 20,05\% a inicios de 2010.

En este contexto nació la penúltima reforma de 2010. Las anteriores reformas laborales habían pretendido con más o menos éxito reducir la temporalidad de los contratos y la tasa de paro, pero esta crisis dejó al descubierto el principal defecto de la normativa que es la gran falta de flexibilidad para adaptarse a diferentes coyunturas y la clara dicotomía laboral existente entre contratos indefinidos muy protegidos y contratos temporales muy desprotegidos. Todo ello ha llevado a que en España la destrucción de empleo sea la más brutal de todos los países de la Unión Europea. Una de las principales medidas es el abaratamiento del despido en todos los contratos indefinidos con una indemnización por despido improcedente de 33 días por año trabajado, hasta un máximo de dos años, y en el caso de contratos temporales el coste pasa de ocho a doce días. También se limita el uso del contrato por obra y servicio a cuatro años máximo y para abaratar más los costes del despido a las empresas el Gobierno subvencionará con cargo a Fogasa ocho días de la indemnización de todos los trabajadores indefinidos. Finalmente la reforma facilita que los ERE temporales se apliquen solo a unas horas de la jornada, mientras que el trabajador cobrará parte del paro.

Con unos niveles de tasa de paro del $20,5 \%$ se llega a la última reforma en febrero de 2012, cuyo principal objetivo es paliar la rigidez que presenta el mercado laboral. Las medidas tomadas van orientadas a reducir la tasa de paro, pero incentivando la contratación indefinida, dilatando los contratos de formación y aprendizaje, e impulsando la utilización del contrato a tiempo parcial. En cambio las medidas para flexibilizar el sistema son de carácter interno, haciendo referencia a la movilidad funcional del trabajador, a la modificación sustancial de las condiciones de trabajo y a la simplificación de la burocracia, entre otras.

Con todo esto, España se posicionó como el país de la UE y OCDE con mayor porcentaje de contratos temporales, lo cual podría ser síntoma de gran flexibilidad, pero lo que se observa es que genera efectos adversos como: a) una extraordinaria volatilidad en el empleo; b) una excesiva rotación de los puestos de trabajo; c) la coexistencia de trabajadores parados de corta duración y los de larga duración; d) un bajo crecimiento de la productividad agregada debido a la segmentación de los puestos de trabajo, los contratos indefinidos y los temporales; y e) una discriminación salarial entre los dos tipos de contratos. A todo esto se debe añadir que los costes por despido desincentivan a la contratación indefinida.

Todo lo expuesto refleja que las numerosas medidas tomadas en el mercado laboral español no han sido suficientes ni para flexibilizarlo, ni para poder adecuarse a las diferentes situaciones económicas como la crisis actual. Por este motivo, Europa propone un cambio de paradigma en el mercado laboral español, apostando por el contrato único laboral, el cual implicaría:

1. Acabar con la dualidad entre contratos temporales e indefinidos.

2. Que las decisiones de contrato/despido dependan en menor medida de la protección relativa de cada trabajador y permitir que el empleador defina plantillas por productividad.

3. Que sea el Estado quien decida el nivel de protección que ofrece a un desempleado. Esto para que la protección ante las inevitables fluctuaciones en la 
economía no condicione las decisiones de las empresas, y el coste de esas fluctuaciones sea asumido entre todos.

4. Simplificar y facilitar la toma de decisiones de personal, para evitar así costes innecesarios.

Dada la situación actual y partiendo del supuesto de que como herramienta para una correcta política de empleo, la legislación laboral debe sentar las bases sólidas para que en el momento de recuperación económica se generen puestos de trabajo estables, se presenta un modelo de simulación de contratación que nos va a permitir un análisis de sensibilidad de las distintas variables por tener en cuenta en una regulación del mercado laboral. Dicho modelo parte del escenario de expectativas de crecimiento por parte del empleador.

El modelo consiste en asimilar el comportamiento del empresario al del propietario de una cartera de inversión compuesta por dos derivados reales. Si entendemos el contrato de trabajo como una transferencia entre empresario y trabajador donde se intercambian producción por salario, se puede definir en términos de derivados financieros como un swap. Por otro lado, el empresario quiere tener la posibilidad de rescindir el contrato laboral cuando el puesto de trabajo no le resulte rentable, es decir, que la producción del empleado sea inferior al salario que percibe, pagándole al trabajador los costes de indemnización que está obligado por ley. Por esto, se interpretar como la compra de una opción de venta americana (american put).

Así pues, el principal objetivo de este trabajo es doble:

1. Determinar la sensibilidad que presentan las diferentes variables por tener en cuenta en la contratación respecto a la decisión de contratar por parte del empresario.

2. Determinar cómo afecta en la decisión de contratar el principal activo de la última reforma laboral, que es la disminución de los días de indemnización por año trabajado.

\section{MODELO DE DECISIÓN DE CONTRATACIÓN A TRAVÉS DE DERIVADOS REALES: REAL DERIVATIVES HIRING DECISION MODEL (RDHDM)}

La aplicación de derivados reales, más concretamente opciones, a la valoración de circunstancias específicas en la toma de decisiones de la política de recursos humanos empresarial ha sido abordada en diversas ocasiones en la literatura. Este es el caso de Sanyal y Sett (2011), cuyo estudio empírico referente a empresas en la India toma como variables explicativas del performance de la empresa, la utilización de determinados tipos de prácticas contractuales asimilables a distintos tipos de opciones reales a los que llaman human resources options.

El enfoque de la teoría de opciones aplicada a la valoración de determinadas características de los contratos laborales también se ve reflejado en el artículo de Bhattacharya y Wright (2004), en el que hacen uso de las opciones reales para incluir la incerteza y el riesgo como factores de decisión en la política de recursos humanos. Foote y Folta (2002), por su parte, toman un enfoque similar al utilizar las opciones reales como medio para incluir la posibilidad de evaluar la irreversibilidad de los contratos laborales fijos respecto a los temporales. La aplicación de derivados reales para determinar la heurística del empresario en decisiones referentes a contratos laborales se aborda en el trabajo de Gete y Porchia (2013) y la decisión de salida del contrato laboral por parte del empleador, analizando las decisiones de despido en un entorno de dualidad en la legislación contractual a partir de la teoría de opciones.

El desarrollo teórico ejecutado por Bou, Hernandez y Linares (2010) sienta las bases del presente artículo. El citado artículo es pionero en utilizar los derivados reales en la determinación de la heurística que el empresario aplica al tomar la decisión de contratar a un trabajador, y considera así un contrato laboral de carácter indefinido como un activo equiparable a una cartera de derivados reales, compuesta por un swap de tipo fijo contra 
tipo variable más una opción de venta, cuyo activo subyacente es el mismo swap.

Este enfoque nos permite utilizar los conceptos de dos derivados financieros para convertir la contratación de un trabajador en una cartera de derivados reales, y así utilizarla para simular el comportamiento del empresario, a distintos incentivos económicos, en el momento de contratar. De este modo, se conseguirá un modelo que, una vez valorado, permitirá simular los efectos de las posibles medidas que podría incorporar la reforma laboral española.

\section{El contrato de trabajo indefinido como un swap}

Un swap es un contrato entre dos partes que se comprometen a intercambiarse flujos de caja (cash-flows) futuros durante un plazo determinado. Concretamente, un swap fijo contra variable es una de las tres modalidades existentes de swaps no exóticos (sin opcionalidad). En esta, una de las partes paga periódicamente un cash-flow constante, es decir, paga los flujos originados por la pata fija (fixed leg) del contrato. En cambio, la otra parte paga periódicamente un cash-flow variable determinado por la evolución del activo subyacente, es decir, paga los flujos derivados por la pata variable (floating leg).

La extrapolación del mercado financiero al mercado laboral resulta al considerar la contratación indefinida de un trabajador como la firma de un contrato de swap fijo contra-variable entre la empresa o empresario (a partir de ahora se consideraran por igual) y el trabajador. Así pues, la empresa o empresario pagará un cash-flow fijo periódicamente durante un plazo determinado, es decir, pagará el salario al trabajador mensualmente hasta su jubilación. A su vez, el trabajador le entregará a la empresa un flujo variable, el valor de su producción mensual.

A continuación se muestran en la tabla 1 y en la gráfica 1 de cash-flows la equivalencia de parámetros entre un contrato de trabajo y un swap de tipo de interés fijo contra-variable básico conocido como "plain vanilla".

\section{Tabla 1.}

Ejemplo comparativo entre un contrato laboral y un plain vanilla

\begin{tabular}{|c|c|c|}
\hline Parámetro & Swap plain vanilla & Contrato laboral \\
\hline Fixed leg & $2 \%$ sobre el nocional & Salario \\
\hline Activo Subyacente & Euribor 1 mes & Producción \\
\hline Fixed leg payer & Contrapartida A & Empresa o empresario \\
\hline Floating leg payer & Contrapartida B & Trabajador \\
\hline Vencimiento & 3 años & Jubilación trabajador \\
\hline Periodicidad & Mensual & Mensual \\
\hline Inicio & $23-09-20010$ & Spot \\
\hline
\end{tabular}

Fuente: elaboración de los autores, con base en datos de Infobolsa. 
Representación gráfica del ejemplo comparativo entre un contrato laboral y un plain vanilla
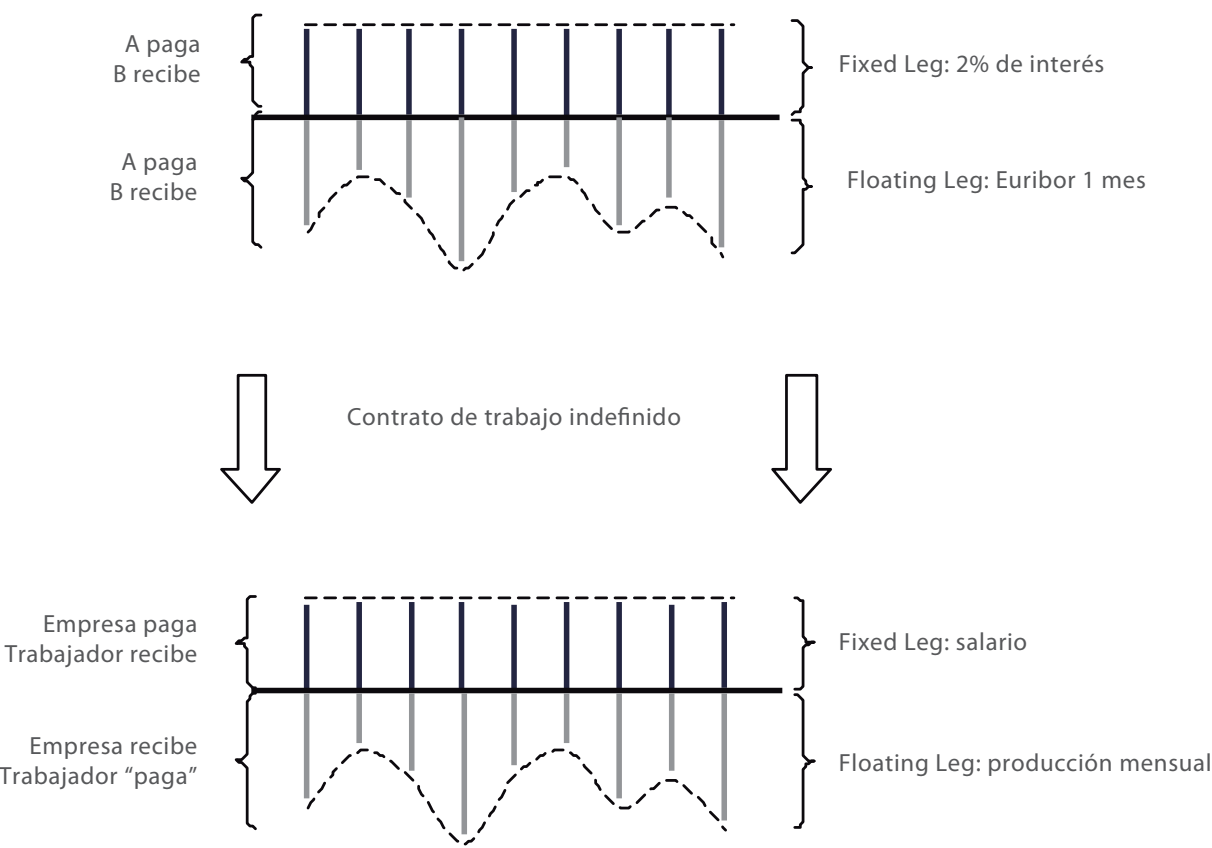

Fuente: elaboración de los autores.

\section{La posibilidad de despido como una opción de venta}

En el momento en el que el empresario analiza la contratación de un trabajador, este tiene en cuenta la posibilidad de llegar a despedir al trabajador si las previsiones que determinaron su contratación no se cumplen, es decir, cerrar su posición en el swap. De este modo, los empresarios adversos al riesgo estarían dispuestos a contratar una cobertura o seguro para eliminar las posibles pérdidas originadas por sus errores de predicción. Siguiendo un comportamiento racional, el empresario exigiría en la contratación un valor inicial adicional del contrato de trabajo para financiar su cobertura. En cambio, los empresarios propensos al riesgo exigirían un valor inicial adicional, como remuneración del riesgo asumido. En ambos casos, el empresario exige un valor adicional al contrato de trabajo igual al de la cobertura. Por este motivo, el valor de una cobertura es la mejor referencia existente para cuantificar el valor de una posible pérdida futura.

En el mercado laboral no existe tal cobertura, pero en el mercado financiero se cotiza un derivado que, adaptándolo a los términos de un contrato de trabajo, se comportará como la cobertura que se está buscando. Este derivado es una opción de venta americana, por lo que una vez que obtengamos el valor de la prima, podremos considerarla como el valor de la posibilidad de despido que el empresario descontará del valor inicial del contrato de trabajo.

Una opción de venta americana o american put es un contrato de derivado financiero entre dos partes en el que el comprador adquiere el derecho pero no la obligación de vender un activo determinado (activo subyacente), dentro de un periodo acordado (en cualquier periodo entre el inicio y el vencimiento) y a un precio establecido (strike). 
En cambio, el vendedor asume la obligación de comprar el activo subyacente, si el comprador ejerce su derecho, a cambio de una prima en el momento de la contratación. El valor de la prima está determinado por el valor inicial y la evolución del activo subyacente, respectivamente, y la volatilidad.

La extrapolación de la opción de venta americana es más complicada y abstracta que la que se ha realizado anteriormente con el swap. Por este motivo, y para facilitar la comprensión, se ha considerado conveniente describir la conversión de la opción financiera por etapas hasta obtener el objetivo perseguido.

1. La posibilidad de despedir a un trabajador es binaria, es decir, la empresa despide o no, de modo que no consideramos la existencia términos intermedios, como la reducción de jornada. Esta posibilidad binaria es igual a la que se enfrenta un comprador de una opción (ejercer su derecho o no), por lo que se considerará que la empresa compra una opción al contratar a un trabajador.

2. El despido del trabajador está motivado por las pérdidas que genera el contrato de trabajo a la empresa, ya que el salario bruto mensual supera el valor de la producción. Por tanto, si existiera, la empresa adquiriría en el momento de contratación una cobertura que le permitiese deshacerse de la producción futura a cambio de las obligaciones de pago futuras a las que se ha comprometido en la firma del contrato de trabajo. Es decir, rescindir el contrato de trabajo, por lo que se considerará que la empresa compra una opción de venta.

3. El empresario no puede predecir con certeza en qué momento se le originará la necesidad de despedir al trabajador, y por su condición de maximizador de beneficios requiere poder hacerlo mensualmente; por esta razón, se considerará que la empresa compra una opción de venta americana que puede ejercer cada mes.

4. La evolución del activo que hace variar el valor de la opción de venta americana es el contrato de trabajo, pero como los costes salariales totales son constantes, podemos afirmar que es la evolución del valor futuro de la producción el activo que determina el valor de la opción. Así pues, se considerará el valor futuro de la producción como activo subyacente de la put americana.

5. Con la firma de un contrato de trabajo, la empresa adquiere un activo a cambio de un pasivo, es decir, la empresa tiene el derecho a la producción del trabajador y la obligación de pagarle. Por ello, en el momento de despido querría vender su derecho a la producción futura por el valor de los costes salariales y así cerrar su posición, es decir, compensar los derechos y obligaciones adquiridos en el contrato de trabajo.

Hay que contemplar que para incrementar la estabilidad laboral el Gobierno establece por ley un coste de despido. Este coste es comparable con una tasa unitaria sobre el precio de venta, como la que se aplica en España a la gasolina, pero con la diferencia de que es asumida totalmente por la empresa. Es decir, cuando la empresa despide a un trabajador se le origina por ley un pasivo adicional al que incurrió en el momento de la contratación.

De este modo, la empresa estaría dispuesta a adquirir un seguro que, aparte de permitirle cerrar su posición en el contrato de trabajo, le cubriera la pérdida generada por el coste de despido y así conseguir una "cobertura perfecta" de sus riesgos. Para ello, la empresa tendría que recibir los costes salariales futuros más el coste de despido cuando ejerciera su derecho de venta sobre la producción futura.

El coste de indemnización funciona como una barrera de salida en el mercado laboral, ya que al incrementar el pasivo del empresario disminuye 
los incentivos económicos sobre la consideración del despido. Indirectamente, este coste podría originar una barrera de entrada al incrementar los riesgos a los que se expone el empresario en un contrato de trabajo, lo cual encarece el coste de la cobertura. Este hecho obligará al empresario a exigir un valor actual adicional a un contrato de trabajo en el momento inicial por asumir el riesgo originado.

En definitiva, se consideraran los costes salariales totales más los costes de indemnización como el strike de la opción de venta americana.

Es necesario definir un horizonte temporal finito para cuantificar correctamente el valor de la producción futura y los costes salariales totales. Teniendo en cuenta que el empresario tiene un horizonte temporal de previsión finito - la visión del empresario-, será este el que se utilice como longitud temporal para determinar ambos flujos. Por ejemplo, si la visión del empresario es de treinta meses, los costes salariales totales futuros serán los salarios y sus respectivas cotizaciones sociales generadas en treinta meses, y el valor de la producción futura será la previsión de treinta meses de producción.

En resumen, el valor de la posibilidad de despido será considerado como la prima de una opción de venta americana con la producción futura como activo subyacente, la visión del empresario como vencimiento y los costes salariales totales futuros más el coste de indemnización como strike.

Como se ha realizado para el caso de swap, se ha resumido en una tabla la equivalencia de parámetros entre la opción de venta americana y la posibilidad de despido.

Tabla 2.

Ejemplo comparativo entre el despido y la opción de venta americana

\begin{tabular}{|ccc|}
\hline Parámetro & American put & Posibilidad de despido \\
\hline Activo subyacente & BBVA.MC & Producción futura \\
\hline Inicio & $10-06-2010$ & Spot \\
\hline Vencimiento & $22-07-2010$ & Visión del empresario \\
\hline Comprador & Agente A & Empresa o empresario \\
\hline Emisor & Agente B & Trabajador \\
\hline Strike & $12,40 €$ & Costes salariales futuros + indemnización \\
\hline Liquidación & Delivery & Por diferencias \\
\hline
\end{tabular}

Fuente: elaboración de los autores, con base en datos de Infobolsa.

\section{METODOLOGÍA}

La valoración de la cartera de derivados propuesta es la siguiente:

\section{Valoración del swap}

La herramienta de valoración de un swap es sencilla y es ampliamente utilizada en otros ámbitos de las finanzas, como en la valoración de proyectos. El swap se valora mediante el valor actual neto y consiste en actualizar los flujos futuros esperados a la tasa de descuento correspondiente. El hecho de utilizar solamente previsiones de flujos futuros simplifica la valoración, ya que para determinar los flujos futuros solo son necesarias las previsiones de producción del empresario, y además permite la valoración ex-ante.

Consideraremos una empresa "tipo", para seguidamente poder generalizar los posibles resultados encontrados. Para ello asumimos los distintos supuestos: 
1. Estructura básica: con el objetivo de facilitar la cuantificación de las previsiones del empresario que pueden determinar la contratación de un nuevo trabajador, se supondrá que la empresa "tipo" se encuentra en un nivel de trabajadores mínimo para satisfacer la demanda actual. Es decir, cualquier previsión de incremento de ventas (o producción) positivo no podrá ser asumida por ningún trabajador actual de la empresa, sino que la empresa deberá contratar a un nuevo trabajador para que pueda producir las posibles previsiones. Es importante remarcar que, después de la recesión vivida en España durante 2009, las empresas han despedido a trabajadores para poder reducir su estructura empresarial al mínimo y así poder sobrevivir a la magnitud de la crisis. Por ello, este supuesto se ajusta a la realidad actual del mundo empresarial, los posibles resultados que se obtendrán a partir del modelo, ya que actualmente muchas empresas se encuentran en situación de estructura básica de empleados.

2. Empresa solvente: como se ha anunciado anteriormente, el objetivo del trabajo se centra en el estudio de la reactivación de la contratación indefinida y estudiar ex-ante las posibles medidas adoptadas por la actual reforma laboral. Este hecho obliga a la imposición del supuesto de que la empresa "tipo" es solvente en el largo plazo y así se podrían descartar las probabilidades de default o quiebra que harían inconsistentes los resultados del estudio empírico.

3. Costes de contratación y costes salariales: los costes de contratación derivados del proceso de selección, trámites administrativos y publicidad son muy diversos a lo largo del tejido empresarial. Además, estos costes originados por las asimetrías de información entre las dos partes del contrato de trabajo pueden ser causantes de ineficiencias en la contratación. Por estos motivos, se ha considerado necesario aplicar el supuesto de la inexistencia de costes de contratación. Para determinar la valoración de los flujos fijos futuros se asumirá que el coste salarial total que paga la empresa está compuesto por las cotizaciones sociales y el salario del trabajador. No se tiene en cuenta la existencia de cláusulas de indización en los contratos, es decir, los salarios no serán ajustados anualmente al Índice de Precios del Consumo (IPC). Aunque gran porcentaje de contratos indefinidos actuales contienen esta cláusula, los diferenciales de inflación en los que se contextualiza el trabajo son relativamente bajos.

4. Previsión de producción: el mecanismo según el cual los empresarios españoles construyen sus previsiones futuras es relativamente complejo y subjetivo a sus características, formación, experiencia, sector al que se enfrentan, etc. Por ello, y para facilitar la construcción del modelo se considerara que las previsiones de producción crecen a una tasa anual constante que, una vez aplicadas a la demanda o producción de la estructura básica de la empresa, permitirá obtener la previsión de producción adicional que debería satisfacer el nuevo trabajador si es contratado.

5. Visión del empresario: esta se entiende como la duración de las previsiones a lo largo del intervalo temporal del estudio. Este horizonte finito determinará el vencimiento en la valoración del contrato de trabajo y de su cobertura. Por la complejidad que originaría su constante modificación, se aplicará el supuesto de que la visión del empresario es constante. Anteriormente, en el proceso de convertir un contrato de trabajo 
en un derivado, se ha considerado la jubilación del trabajador como la fecha de vencimiento del contrato indefinido. Pero debido a que el empresario tiene la posibilidad de rescindir el swap en cualquier mes y la volatilidad asociada a unas previsiones de tal longitud temporal, este considera periodos inferiores en el momento de la contratación (la visión del empresario). Las previsiones de producción están directamente relacionadas con la demanda de producto que el empresario prevé que recibirá la empresa en el futuro, por lo que se consideran por igual las previsiones de producción que las de demanda. En ninguna se contempla el diferencial de inflación anual.

6. Tasa de descuento: estas tasas se consideran constantes en el tiempo.

7. Trabajador: el trabajador que se considera para la contratación es perfectamente sustituible por otro candidato, ya que no dispone de cualidades especiales ni de un nivel de capital humano elevado.

Teniendo en cuenta estos supuestos, el valor actual del contrato de trabajo (VACT) queda formulado del siguiente modo:

$$
V A C T=\frac{\sum_{\tau=0}^{\tau} \alpha_{\tau}}{(1+f)^{\tau}}-\frac{\tau * \omega(1+s)}{\left(1+i_{12}\right)^{\tau}}
$$

Donde las variables son:

$\alpha$ : previsión de la producción en cada mes que tendría que satisfacer el nuevo trabajador, es decir, los flujos variables previstos en el futuro.

$f$ : tasa de descuento aplicado a las previsiones; como estas tienen riesgo de que no se cumplan, su tasa de descuento debe ser mayor que la tasa de descuento libre de riesgo. $\tau$ : visión del empresario, es decir, cuántos meses futuros influyen en la decisión del empresario en el momento de contratar.

$\omega$ : salario del trabajador.

s: porcentaje de cotizaciones sociales por aplicar al salario.

$i_{12}$ : tasa de descuento libre de riesgo efectiva mensual

\section{Valoración de la opción de venta americana}

En la teoría financiera existen dos grandes métodos para valorar opciones. Estos son el modelo de Black-Scholes y la técnica del árbol binomial o Binomial Option Pricing Model (BOPM). Ambos métodos llegan a converger en la valoración de opciones europeas, pero debido a la necesidad de contemplar ejercicios anticipados (opción americana), es decir, despidos antes de que finalice la visión del empresario, se ha elegido la técnica del árbol binomial desarrollada por Cox, Ross y Rubinstein (1979), y adaptada por John C. Hull (2009)

Para asegurar la consistencia de los resultados obtenidos en la valoración de la opción de venta americana, es necesario realizar los siguientes supuestos:

1. Distribución del valor futuro de la producción: en el momento en el que el empresario se plantea contratar se basa en las previsiones de producción (o demanda) que le abastecerá el nuevo trabajo. Cuando se han formulado los supuestos de valoración del VACT se ha asumido que las previsiones crecen a una tasa constante. La suma de las previsiones mensuales dentro de la visión del empresario (valor de la producción futura) son modificadas a lo largo del tiempo, ya que el empresario recibe nueva información del mercado de producto o servicios en el que compite. Con el objetivo de extraer resultados consistentes a partir del BOPM, es 
necesario establecer el supuesto de distribución binomial en la sucesión de valores futuros de producción posterior a partir del momento de contratación. Esta distribución converge con la distribución normal a partir de los treinta periodos (en este estudio, meses).

2. Valuación neutral al riesgo: la valuación neutral al riesgo es uno de los supuestos básicos del modelo BOPM. Si se extrapola este supuesto al ámbito en el que se contextualiza el trabajo, consistiría en considerar que los empresarios no requieren ninguna compensación por el riesgo, y el rendimiento esperado de la producción futura sería la tasa libre de riesgo. Es decir, estos principios establecen la asunción de que el mundo es neutral al riesgo al valorar opciones. El empresario realiza sus previsiones de producción futura, desde el momento inicial del estudio, a partir de una tasa constante y así consigue una producción esperada cada mes. En cada momento del tiempo futuro analizado se suman el valor de las previsiones de producción dentro de una longitud temporal igual a la visión del empresario y así se obtiene el valor de la producción futura en el mes $t$. Con el fin de poder realizar la valoración de la opción, se asume que el rendimiento de la producción futura es la tasa libre de riesgo.

\section{El árbol binomial como herramienta de valoración}

Un árbol binomial es un diagrama que representa las diversas trayectorias que puede seguir el valor de la producción futura durante la visión del empresario. Es decir, en el momento en el que el empresario se plantea la contratación, proyecta a partir de la producción futura esperada en ese momento y durante un periodo igual a la "visión

Finanz. polit. econ., ISSN 2248-6046, Vol. 6, No. 1, enero - junio, 2014, pp. 73 - 94 Edición Especial - Economía Regional del empresario" todas las posibles producciones futuras de la misma longitud temporal que esperará en cada mes del periodo de la opción hasta su vencimiento.

Para diseñar el árbol binomial que represente correctamente el comportamiento de la producción futura en un mundo neutral al riesgo es necesario que los siguientes parámetros proporcionen los valores correctos de $p$, u y d para que la media y la varianza del rendimiento del valor de la producción futura durante un intervalo $\Delta \mathrm{t}$. Dichos parámetros son los siguientes:

- La probabilidad "p" de que el valor de la producción futura aumente.

- La probabilidad inversa "1-p" de que el valor de la producción disminuya.

- El factor " $u$ " que determine el valor de la producción futura si esta aumenta, por lo que deberá ser mayor a uno.

- El factor " $d$ " que determine el valor de la producción futura si esta disminuye, por lo que deberá ser inferior a uno.

- De este modo, se iguala el rendimiento promedio del valor de la producción futura con el árbol binomial se necesitaría:

$\alpha \cdot e^{i \Delta t}=p \cdot \alpha \cdot u+(1-p) \cdot \alpha \cdot d$

Al dividir por $\alpha$, se obtiene el valor de producción futura en el inicio del intervalo:

$e^{i \Delta t}=p \cdot u+(1-p) \cdot d$

Una vez se obtiene el rendimiento promedio, se establece la varianza del rendimiento del valor de la producción futura en un pequeño intervalo $\Delta \mathrm{t}$ como $\sigma 2 \Delta \mathrm{t}$, donde $\sigma$ es la volatilidad de la demanda. Aplicando la definición de la variancia se deduce que: 
$\sigma^{2} \cdot \Delta t=p \cdot u^{2}+(1-p) \cdot d^{2}-$

$[p \cdot u+(1-p) \cdot d]^{2}$

Finalmente, cuando $\Delta \mathrm{t}$ es pequeño, y teniendo en cuenta la condición impuesta por Cox, Ross y Rubinstein ( $u=1 / d)$, las dos ecuaciones anteriores se resuelven por medio de:

$p=\frac{e^{r \Delta t}-d}{u-d}$

$u=e^{\sigma \sqrt{\Delta t}}$

$d=e^{-\sigma \sqrt{\Delta t}}$
En el árbol binomial adaptado para determinar las proyecciones de la producción futura se ha considerado que el empresario modifica sus previsiones de producción futura mensualmente de manera discreta, por lo que el $\Delta$ t mínimo será un mes.

Teniendo en cuenta todo lo explicado, ya se puede elaborar un árbol binomial que determine correctamente los posibles valores de la producción futura. Con la finalidad de resumir la construcción de un árbol binomial, se muestra el proceso de cálculo asumiendo una longitud temporal de dos meses, donde cada recuadro de éste representa un nodo (nt, j):

\section{Determinación del precio de ejercicio}

El precio de ejercicio al que el empresario estaría interesado en vender el contrato de trabajo está compuesto por los costes salariales totales futuros, determinados por la visión del empresario y por el coste de indemnización. Según la legislación actual, el coste de despido crece a una tasa constante en el tiempo que es medida en días

Representación gráfica para dos periodos del árbol binomial utilizado.

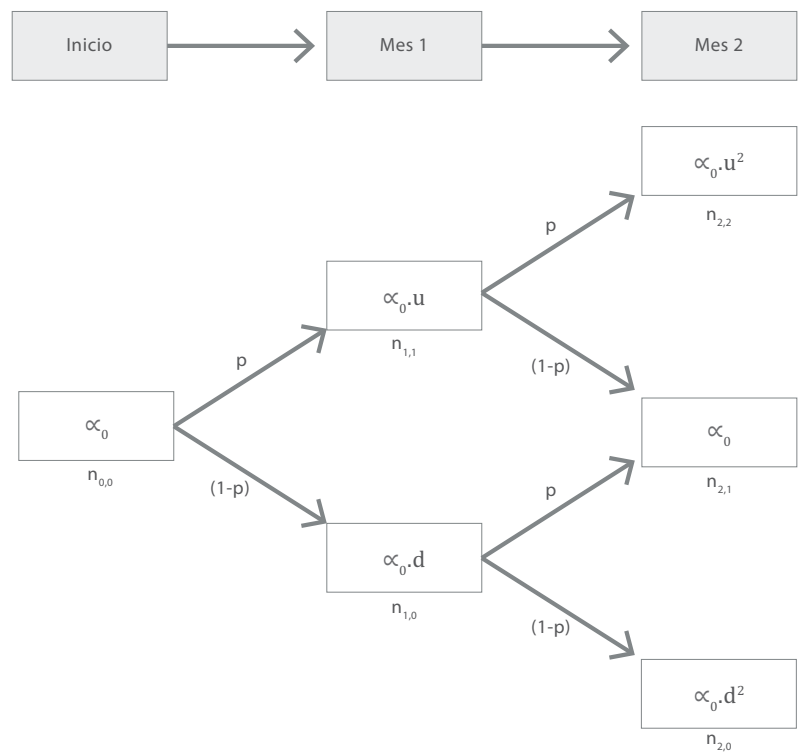

Fuente: elaboración de los autores. 
de salario por año trabajado. Por este motivo, el precio de ejercicio no es una constante, sino que, es una función lineal creciente en el tiempo que algebraicamente se representa como:

$$
\text { Strike }_{t}=v \cdot w \cdot(1+s)+w \cdot \frac{m}{360} \cdot t
$$

Donde las variables son:

$\checkmark$ visión del empresario.

$w$ salario bruto que percibe el trabajador por parte de la empresa

$s$ porcentaje de cotizaciones sociales por aplicar al salario

$m$ tasa de crecimiento del coste de indemnización en términos de día de salario por año trabajado. Se divide entre 360 como factor de conversión para así obtenerla en términos mensuales.

$t$ mes que representa el nodo del árbol binomial, siendo $v$ el valor máximo.

\section{Valoración de la opción de venta americana}

El procedimiento de valoración de una opción cuando el árbol binomial está creado es relativamente sencillo e intuitivo. Es conocido como inducción hacia atrás o backwards inductions y consiste en valorar la opción en las distintitas proyecciones de la producción futura en cada momento del tiempo desde el vencimiento hasta el inicio. Empezando por los nodos del árbol binomial pertenecientes al vencimiento, se calcula para cada nodo el valor de la opción que será el valor máximo entre el beneficio de ejercer el derecho de vender o no ejercer.

$$
P u t_{v, j}=\operatorname{Max}\left(\text { strike }_{v}-\alpha_{0} \cdot u^{j} \cdot d^{v-j}\right) ; 0
$$

Para calcular el valor de la opción en los demás nodos siguiendo el proceso de inducción hacia atrás para luego llegar al valor en el momento cero (prima), se ha de considerar en la fórmula anterior la posibilidad de que el valor de mantener la opción otro periodo más sea mayor al beneficio de ejercer. Es decir, el valor de opción en estos nodos será el máximo entre el beneficio de ejercer, no ejercer y el valor intrínseco de la opción. Si $\alpha i, j$ es el valor de la producción futura en el nodo, el valor de la opción algebraicamente es:

$$
\begin{aligned}
& P u t_{t, j}=\operatorname{Max}\left\{\text { strike }_{t}-\alpha_{0} \cdot u^{j} \cdot d^{t-j} ; e^{-i_{12}}\right. \\
& \left.\left[p \cdot \alpha_{i+1, j+1}+(1-p) \cdot \alpha_{i+1, j}\right]\right\}
\end{aligned}
$$

Con el fin de ilustrar la valoración de la posibilidad de despido se realiza el siguiente ejercicio:

Producción futura: $3200 €$

Salario: $1000 €$

Cotizaciones sociales: $25 \%$

Volatilidad anual de la demanda: $20 \%$

Coste de despido: 30 días por año trabajado

Visión del empresario: 3 meses

Tasa de interés libre de riesgo mensual: $1 \%$

Los recuadros del valor de la opción con el borde rojo reflejan que el comprador ejerce su derecho, es decir, la empresa despide al trabajador. En el mes dos podemos ver un ejercicio anticipado característico de las opciones americanas.

En este ejemplo, el valor de la posibilidad de despido es de 219,5, que el empresario debe descontar del VACT.

\section{RESULTADOS I: ANÁLISIS DE SENSIBILIDAD}

Para realizar el modelo desarrollado se ha utilizado un simulador de contratación, software informático que permite determinar cuándo el empresario decidirá contratar un nuevo trabajador y analizar la sensibilidad de la contratación a las variables del mercado laboral. El contexto empresarial considerado para el análisis de una pyme, 
ANÁLISIS DE LA HEURÍSTICA EN LA CONTRATACIÓN EMPRESARIAL A TRAVÉS DE UNA CARTERA DE DERIVADOS REALES

Figura 3.

Representación gráfica de la valoración del árbol binomial para el ejemplo descrito
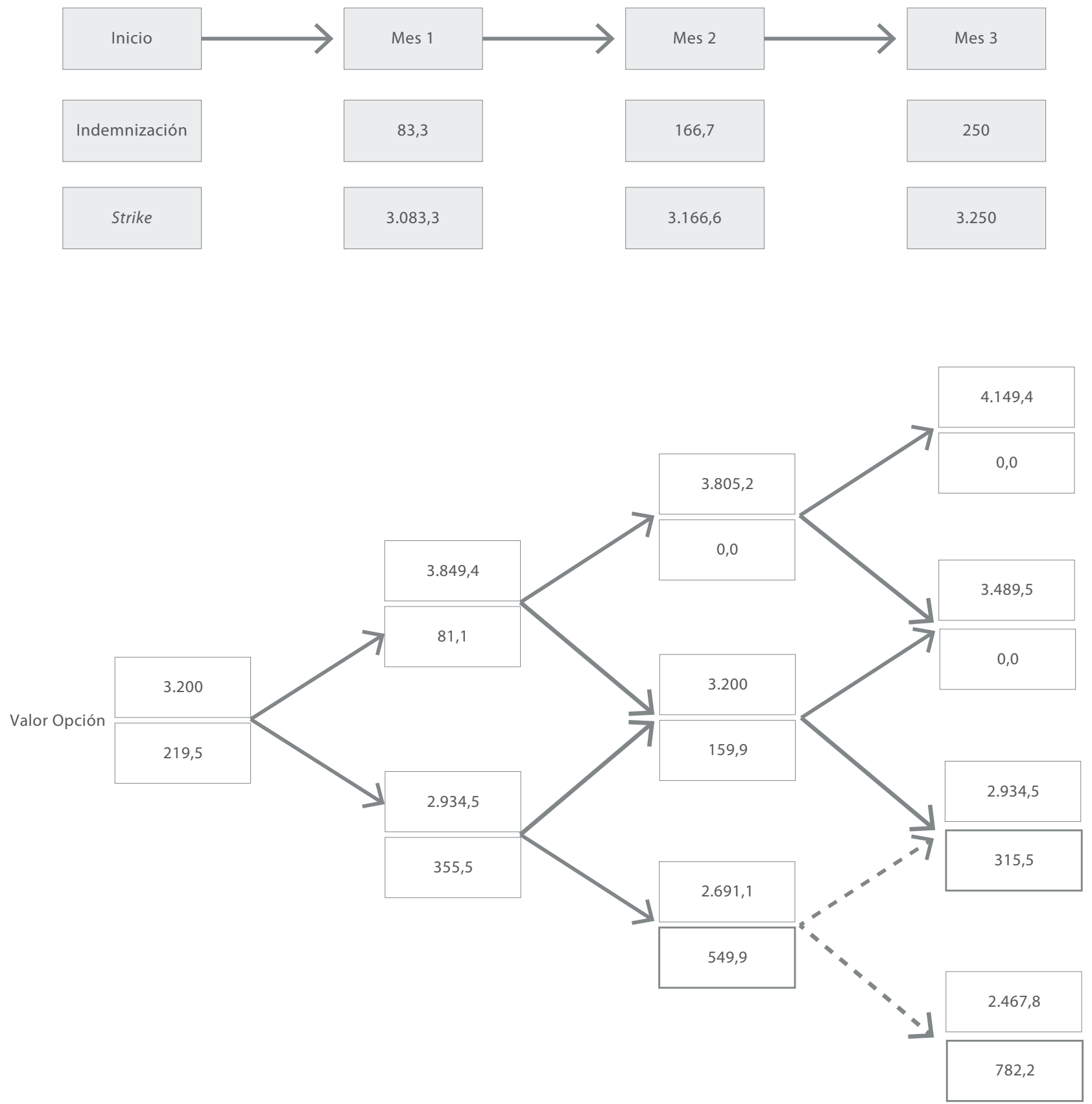

Fuente: elaboración de los autores. 
representativa del tejido empresarial español, y el escenario es la situación económica actual. Las variables de interés que se han supuesto son las siguientes:

1. Demanda anual cubierta por la estructura básica de la empresa: 300.000€, entendiéndola como el volumen de negocio anual que factura una empresa pyme de 15 trabajadores.

2. Crecimiento anual esperado de la demanda: $2 \%$. Representa un incremento modesto que recoge la leve recuperación de la economía española en los próximos años.

3. Visión del empresario: 12 meses. En el escenario de principio de la recuperación económica por estudiar se considera una visión relativamente corta, ya que la difícil situación del tejido empresarial español puede impedir predicciones fiables de largo plazo.

4. Costes variables no salariales por $€$ facturado: $80 \%$. Por esta variable se entienden los costes adicionales derivados de producir una unidad adicional, excepto los del factor trabajo; ya que así se podrá determinar la previsión de producción que tendrá que abastecer el nuevo trabajador si es contratado.

5. Tipo de interés anual: Euribor mayo 2013 $(0,487 \%)$.

6. Factor de descuento de las previsiones: $30 \%$, representando la desconfianza del empresario en las perspectivas del mercado.

7. Volatilidad anual de la demanda: $30 \%$. Esta muestra la variabilidad de la demanda de un año a otro y la incertidumbre característica del momento sobre las futuras ventas, influenciada por el descenso que realizó la demanda interna española a causa de la crisis.

8. Salario bruto mensual que percibe el trabajador: $1000 €$. Teniendo en cuenta que el salario mínimo interprofesional (SMI) está alrededor de los 645,30€, normalmente aplicado a los contratos temporales, se ha considerando un salario más elevado representativo de un contrato indefinido.

9. Carencia: 0 meses, es decir, el empleado empieza a trabajar en la empresa sin período de prácticas o prueba inicial, por lo que en caso de despido los costes de indemnización se computarán desde el primer día de trabajo.

10. Coste de despido: 33 días por año trabajado. Se considera que en caso de despido la naturaleza será improcedente. Como se establece en el Estatuto de los Trabajadores, el trabajador tendrá derecho a percibir 33 días por año trabajado hasta un máximo de 24 mensualidades.

11. Cotizaciones a la seguridad social: $28,3 \%$ tasa aplicada a las contingencias comunes según la Ley de Presupuestos Generales del Estado para 2013.

12. Se considera un contrato indefinido a tiempo completo, dado que es el objetivo por incentivar del Gobierno.

Una vez descritos los parámetros de la situación actual, se puede llevar a cabo el estudio de sensibilidad, es decir, analizar la elasticidad de la contratación respecto a las variables susceptibles de ser modificadas con una nueva reforma laboral orientada al contrato único.

\section{Cotizaciones sociales}

Por un lado, se analiza la sensibilidad a las cotizaciones sociales; es decir, se cómo varía la decisión de contratar a cambios en las cotizaciones que debe pagar el empresario, caeteris paribus. La gráfica 4 muestra la elevada sensibilidad de la decisión del empresario a variaciones de las cotizaciones sociales. Muestra de ello es la distancia temporal que se produce en la contratación si las cotizaciones son nulas (el empresario decide contratar en el mes 7) o si son del $50 \%$ (el empresario contrata en el mes 13). 
Representación de la sensibilidad de las cotizaciones sociales

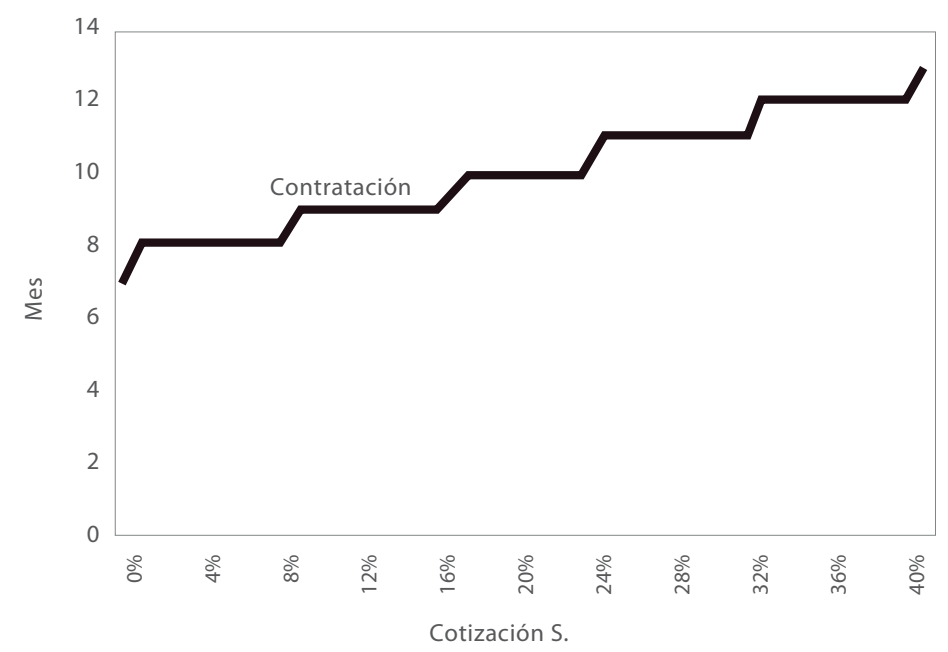

Fuente: elaboración de los autores.

\section{El coste de indemnización}

Se analiza la sensibilidad de la contratación indefinida a los costes de indemnización. A diferencia de lo que en un principio se podría pensar sobre el peso de los costes de despido en la decisión del empresario, se puede comprobar cómo la variación de estos no influye en la contratación de nuevos empleados. Esto se debe, entre otras cosas, al hecho de que la visión del empresario es limitada en el tiempo, solo tiene en consideración 12 meses; por esta razón, los costes de indemnización son

\section{Gráfica 2.}

Representación de la sensibilidad de los días de indemnización

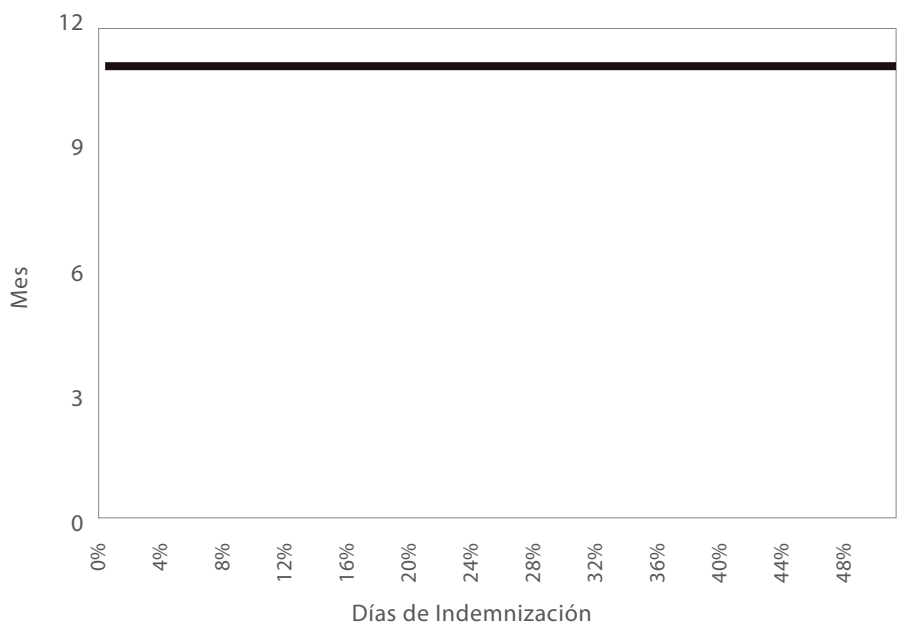

Fuente: elaboración de los autores. 
calculados sobre un año de trabajo, y además cabe la posibilidad de que estos costes no se originen. El valor de estos costes varía muy poco según el nivel de protección del puesto de trabajado, siendo las diferencias no lo suficientemente importantes como para afectar a la decisión del empresario.

\section{El periodo de carencia}

Esta variable se entiende como el plazo inicial en el que el trabajador no acumula antigüedad para los costes de indemnización, a mayor número de meses de carencia en el cómputo de los costes de indemnización, mayor contratación. Aunque se debe remarcar que la sensibilidad de la contratación a esta variable es muy débil, el incentivo a la contratación permanece fijo.

\section{El factor de descuento de las previsiones}

Finalmente, el factor de descuento de las previsiones a causa de la situación económica, aunque no sea susceptible de modificación por parte de los agentes económicos, se puede impulsar indirectamente a partir de medidas que promuevan el crecimiento económico. Como se ve en la gráfica 7, la contratación es muy sensible al factor de descuento que aplica el empresario a las previsiones futuras, debido a la falta de confianza en la evolución de la economía; prueba de ello es el desfase en el tiempo que experimenta la contratación. En el escenario de un $0 \%$ de descuento sobre las previsiones futuras, el empresario decide contratar en el mes ocho, mientras que en otro escenario con una visión pesimista de las perspectivas futuras de la economía (50\% de descuento) contrata cinco meses más tarde.

Gráfica 3 .

Representación de la sensibilidad de la carencia de indemnización

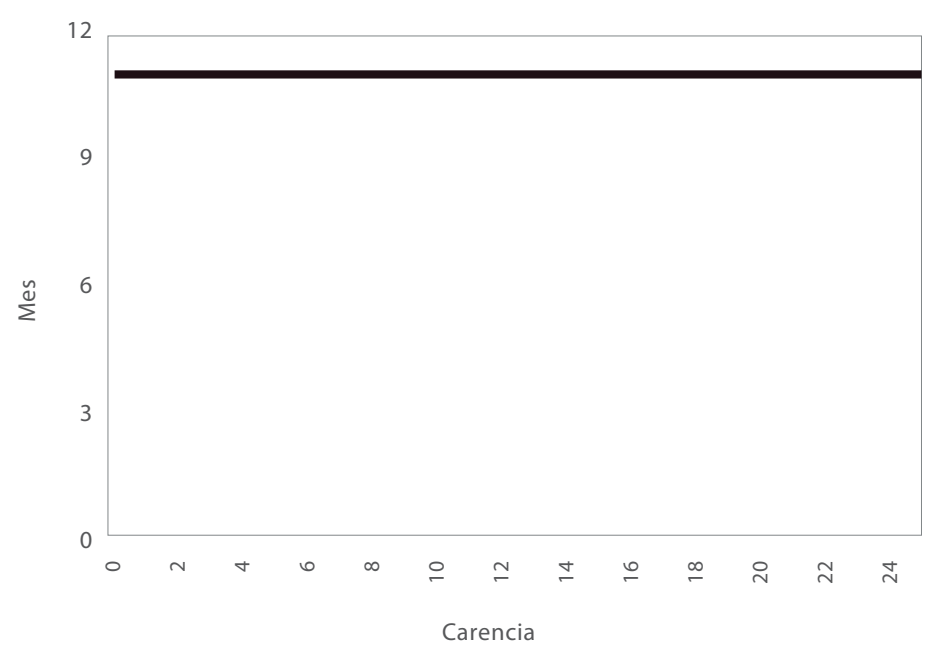

Fuente: elaboración de los autores. 
Representación de la sensibilidad del factor de descuento de las previsiones

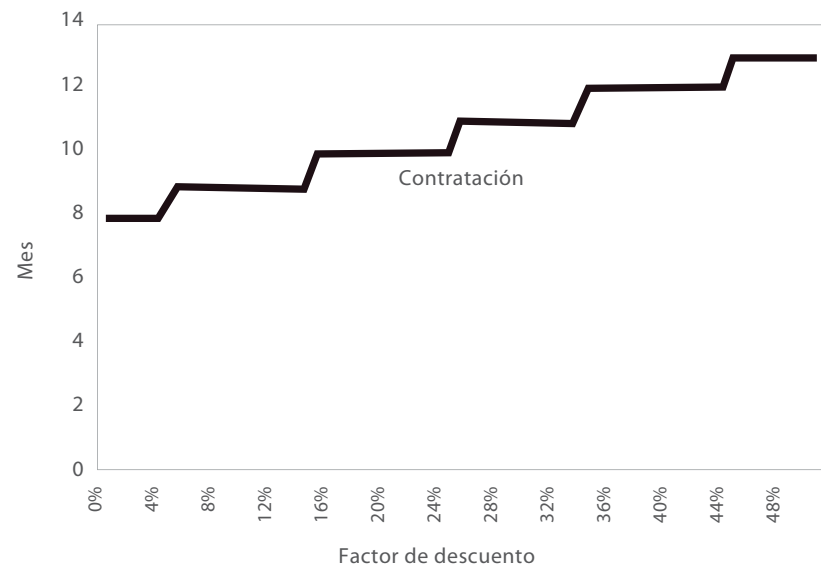

Fuente: elaboración de los autores.

A modo de resumen, se presentan los principales resultados de sensibilidad.

\begin{tabular}{|c|c|}
\hline \multicolumn{2}{|c|}{ Sensibilidad de la decisión de contratar } \\
\hline Cotizaciones sociales & Muy alta \\
\hline Coste de indemnización & Casi nula \\
\hline Período de carencia & Casi nula \\
\hline Tasa de descuento de las previsiones & Muy alta \\
\hline
\end{tabular}

Fuente: elaboración de los autores.

\section{RESULTADOS II: DECISIÓN DE CONTRATACIÓN}

Tras evaluar la sensibilidad de la contratación de las principales variables del simulador en el contexto actual, a continuación se estudia en qué mos para su comparación: mento el empresario toma la decisión de contratar.

\begin{tabular}{|c|c|c|}
\hline & Indemnización (días/año trabajado) & Cotización a la seguridad social \\
\hline Escenario 1 & 45 & $28,30 \%$ \\
\hline Escenario 2 & 33 & $28,30 \%$ \\
\hline Escenario 3 & 33 & $20 \%$ \\
\hline
\end{tabular}

Fuente: elaboración de los autores.

Escenario 1: anterior legislación laboral. Escenario 2: actual reforma laboral, en la cual se reducen los días de indemnización por año trabajado.

Escenario 3: actual reforma laboral, pero incluyendo una posible reducción en las cotizaciones a la seguridad social.
Aplicando el modelo presentado en este trabajo, los resultados son los siguientes:

Gráficas 8, 9 y 10: Representación gráfica de los meses en que el empresario contrata en los tres escenarios planteados 
La decisión de emplear un nuevo trabajador se lleva a cabo en el mes once.

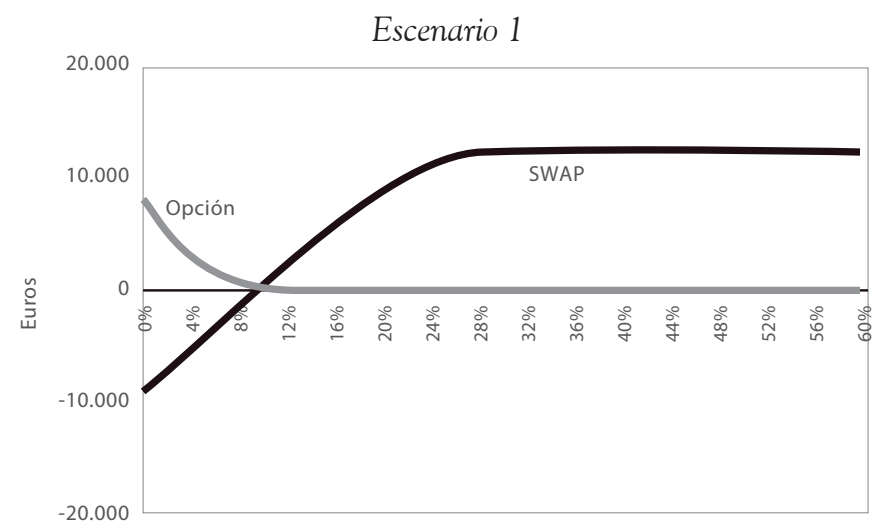

La decisión de emplear un nuevo trabajador

se lleva a cabo en el mes once.

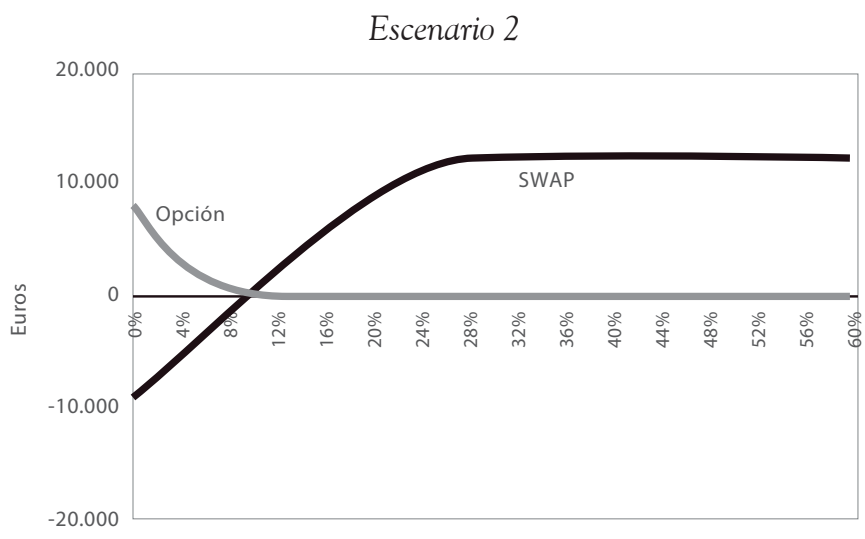

La decisión de emplear un nuevo trabajador se lleva a cabo en el mes diez.

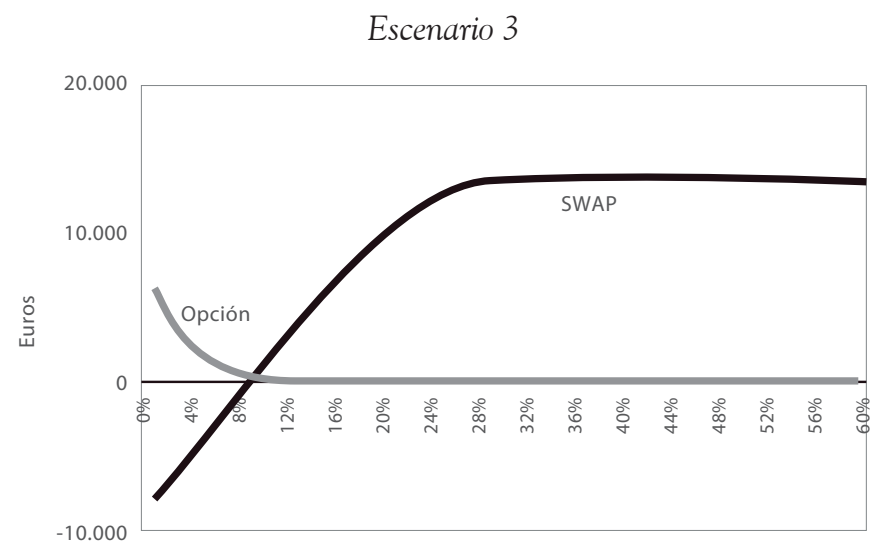


Como se observa en las gráficas anteriores:

1. No existe diferencia en la decisión de contratación a pesar de haber disminuido los días de indemnización por año trabajado. En ambos casos el empresario contrata en el mes 11.

2. Sí existe diferencia en la decisión de contratación cuando a pesar de mantener los días de indemnización se disminuye la cuota patronal a la seguridad social. En este caso la contratación se produce un 1 mes antes que en el caso anterior.

\section{CONCLUSIONES}

Este trabajo realiza un desarrollo teórico basado en una cartera de derivados reales para determinar la heurística que un empleador sigue cuando debe tomar la decisión de contratar a un trabajador.

El modelo resultante permite mediante una metodología de simulación, por un lado, un análisis de sensibilidad de la decisión de contratar con respecto a las distintas variables sobre las que el regulador puede legislar $y$, por el otro lado, la determinación del impacto en la aceleración de la contratación laboral, que resulta de la implementación de la reciente reforma laboral española.

Las variables analizadas en el estudio de sensibilidad son las cotizaciones sociales, los días de indemnización por año trabajado, el periodo de carencia en el cómputo de los costes de indemnización y el factor de descuento de las previsiones.
La simulación muestra una muy alta sensibilidad de la aceleración en los contratos a una reducción en las cotizaciones sociales por realizar por parte del empresario. El efecto sobre la aceleración de los contratos de la reducción de los costes de indemnización, así como del periodo de carencia en el cómputo de estos costes, es casi nulo.

El impacto del factor de descuento de las previsiones del empresario es muy alto.

Estos resultados llevan a concluir que el regulador tiene una herramienta de gran poder acelerador de la contratación en la reducción de las aportaciones del empresario en concepto de cotizaciones sociales. También se concluye que las intervenciones en el sentido de reducir la indemnización en caso de despido o retrasar en el tiempo el derecho a percibirla no tienen efecto en la aceleración de la decisión de contratar por parte del empleador. No por obvio es menos relevante que uno de los factores clave en la aceleración de la contratación quede fuera de la capacidad del regulador ya que viene determinado por las expectativas de crecimiento económico del empresario (factor de descuento).

Al realizar el estudio del impacto en la aceleración de la contratación de la vigente reforma laboral, se concluye que al actuar solamente sobre el coste de despido y no sobre la reducción de las cotizaciones sociales, la aceleración de la contratación y, por tanto, la creación de empleo va a depender enteramente de las expectativas de crecimiento que el entorno económico sea capaz de generar en el empresario. 


\section{REFERENCIAS}

1. Battacharya, M. and Wright, P. M (2004). Managing Human Assets in a Uncertain World: Applying Real Options Theory to HRM. CAHRS Working Paper Series, Cornell University ILR School.

2. Bou, S., Hernández, A. y Linares, C. (2010). Los derivados financieros como herramienta para evaluar la reforma laboral: una aproximación binomial. Working Paper Series, Business Department, Universitat Autònoma de Barcelona.

3. Cox, J.C, Ross, S.A. and Rubinstein, M. (1979). Option Pricing: A Simplified Approach. Journal of Financial Economics, 7(3), 229-263.

4. Gete, P. and Porchia, P. (2013). A Real Options Analysis of Dual Labor Markets and the Single Labor Contract. Working paper Available at SSRN: http://ssrn.com/abstract=1942048 or http://dx.doi.org/10.2139/ssrn.1942048

5. Foote, D.A. and Folta, T.B. (2002). Temporary workers as real options. Human Resource Management Review, 12, 579-597.

6. Gómez, S., Contreras, I. y Gracia, L. (2008). Las reformas laborales en España y su impacto real en el mercado de trabajo en el período 1985-2008. Documento de investigación DI- 764, Cátedra Seat de Relaciones Laborales, IESE Business School.

7. Hull, J.C. (2003). Options, futures, $\mathscr{G}$ other derivates. New Jersey: Prentice-Hall International.

8. Sanyal, S. and Sett, P. K (2011). Applying real options theory to HRM: an empirical study of IT software firms in India. The International Journal of Human Resource Management, $22(01), 72-102$. 